\title{
Optical Tweezers and Their Applications in Biomedical Engineering
}

\author{
Liangmin Zhang* \\ Department of Physics, Physical Sciences, and Geology, California State University-Stanislaus, USA
}

Submission: July 9, 2019; Published: February 27, 2020

*Corresponding author: Liangmin Zhang, Department of Physics, Physical Sciences, and Geology, California State University-Stanislaus, Turlock, California 95382, USA

Abstract

Optical tweezers is a tool based on strongly focused laser beam trapping force. The force of a laser beam originates from the gradient of electric field containing in the focused laser beam. Laser tweezers has been used increasingly as a unique technique for optical trapping, manipulation, and characterizations of biological cells and dielectric micro- and nano-scale particles. It can also be used to make quantitative measurements of living cells.

Keywords: Optical tweezers; Optical trapping; Nano-manipulation

\section{Introduction}

\section{Physics origins of optical trapping force}

Optical tweezers may also be referred to optical trapping. A strongly focused laser beam has the ability to catch and hold particles or a fiber in a size range from nanometers to microns. This technique makes it possible to study and manipulate particles like small dielectric spheres and biological cells [1-3]. The technique of using a focused laser beam as an atom trap was first proposed in 1970 at Bell laboratories [4]. According to physics, when a laser beam is focused through a microscopic objective, each photon will exert force in a single direction. There are two types of forces, the gradient and scattering forces, which exert themselves on a particle and trap it in the path of the laser beam [5]. The gradient force is a result of the electric field gradient present when a laser beam is focused and photons are aligned. This electric field is strongest at the narrowest part of a focused beam, the beam waist. The particle to be studied in the optical trap becomes attracted to the electric field gradient of the beam waist $[4,5]$. The second force affecting dielectric particle movement, the scattering force, is due to the change in momentum experienced by photons traveling in the direction of beam propagation. This force slightly displaces the trapped particle downstream from its original position at the center of the beam waist $[4,5]$. As a result of the combination of both the scattering and gradient force, a particle will be trapped in the optical tweezers apparatus slightly downstream of the laser beam waist. The laser beam used to operate the optical tweezers apparatus maintains a Gaussian profile. This means that the effect of each photon on the trapped particle is dependent upon its distance from the particle. The photons near the beam waist and trapped particle, which exert an attracting force and pull the particle towards the center of the beam, will have a more intense effect than those photons further from the trapped bead attempting to displace the particle from the beam waist [5]. When these distant scattering forces attempt to move the trapped particle to the left or right of the beam center, the high intensity attraction force overcomes this displacement and maintains the particle at the steady position for characterizations of the particle.

\section{Applications of Optical Tweezers}

Optical tweezers was initially designed as an atom or molecule trap. However, it has been found a wide range of applications in biological sciences, nanotechnology, and bioengineering areas.

\section{Application in biological sciences and bioengineering}

The first application of optical trapping in biology was on the trapping of viruses and bacteria [6]. Using a laser beam, it can trap bacteria cells without damaging them. Starting from this experiment, researchers proceeded to trap a variety of cells, including pigmented red blood cells, single-stranded DNA, double-stranded DNA, protein, green algae, diatoms, amoebas, and other protozoans $[1,7,8]$. Further work used scallion cells to create an "artificial cytoplasmic filament" by using the trap to pull a filament from the surface of the nucleus of the cell into the central vacuole of the cell was also performed. 


\section{Applications in chemistry and nanotechnology}

In addition to capturing particles, optical tweezers has also been widely used in the field of nanotechnology. Optically induced torques of optical tweezers can be used as a micro-machine to rotate $\mu \mathrm{m}$-sized anisotropic particles and to arrange them into nanopatterns [8-9]. An optical tweezers array has been made using a computer-designed hologram by projecting collimated TEM00 laser beam onto a diffraction grating to create multiple beams that go through an objective lens to generate multiple optical traps to construct an optical trap array. The optical array can be used to assemble two-dimensional and three dimensional nanoparticle patterns [10]. In studies of colloidal science, direct measurements using optical tweezers showed that an attractive force can exist between like-charged particles in a colloidal suspension near a surface, contrary to theory. Based on this work, metastable colloidal crystals were created [7].

\section{Conclusions and Perspectives}

As a rapidly developing research field, optical tweezers generate exciting possibilities for bioengineering, bioscience, and nanotechnology. In this paper, the applications and principle of optical tweezers are briefly introduced. In the future, various optical tweezers can be built and employed. The controllability and flexibility of optical tweezers can be used to generate nanoand microdevices. The development of optical tweezers has been extended to investigate biological process in vivo. Although the measurement and operation of living organisms in vivo have some challenge and difficulty, optical tweezers can no doubt provide numerous convenience for characterizing the dynamics of living organisms.

\section{References}

1. Choudahary D, Mossa A, Jadhav M, Cecconi C (2019) Bio-molecular applications of recent developments in optical tweezers. Biomolecules 9 (23): 1-19.

2. Polimeno P, Magazzu A, Lati MA, Patti F, Sajia R, et al. (2018) Optical tweezers and their applications. J Quantitative Spectroscopy \& Radiative Transfer 218:131-150.

3. Zhang L, Wang YX, Zhang F, Claus RO (2006) Observation of whisperinggallery and directional resonant laser emission in ellipsoidal microcavities. J Opt Soc Am B 23(9): 1793-1800.

4. Ashkin A (1970) Acceleration and trapping of particle by radiative pressure. Phys Rev Lett 24(4): 156-159.

5. Ashkin A (1997) Optical trapping and manipulation of neutral particles using lasers. Proc Natl Acad Sci 94(5): 4853-4860.

6. Ashkin A, Dziedzic JM (1987) Optical trapping and manipulation of viruses and bacteria. Science 235(3): 1517-1520.

7. Ashkin A, Dziedzic JM, Bjorkholm JE, Chu S (1986) Observation of a single beam gradient force optical trap for dielectric particles. Opt Lett 11(3): 288-290.

8. Liu J, Li Z (2018) Controlling mechanical motion of microparticles in optical tweezers. Micromachines 9(232): 1-28.

9. Gordon JP (1973) Radiation forces and momenta in dielectric media. Phys Rev A 8(1): 14-21.

10. Grier DG (2003) A revolution in optical manipulation. Nature 424(8): 810-816.

\section{Your next submission with Juniper Publishers} will reach you the below assets

- Quality Editorial service

- Swift Peer Review

- Reprints availability

- E-prints Service

- Manuscript Podcast for convenient understanding

- Global attainment for your research

- Manuscript accessibility in different formats

( Pdf, E-pub, Full Text, Audio)

- Unceasing customer service

Track the below URL for one-step submission https://juniperpublishers.com/online-submission.php 